\title{
Artistic expressions of 21st-century change
}

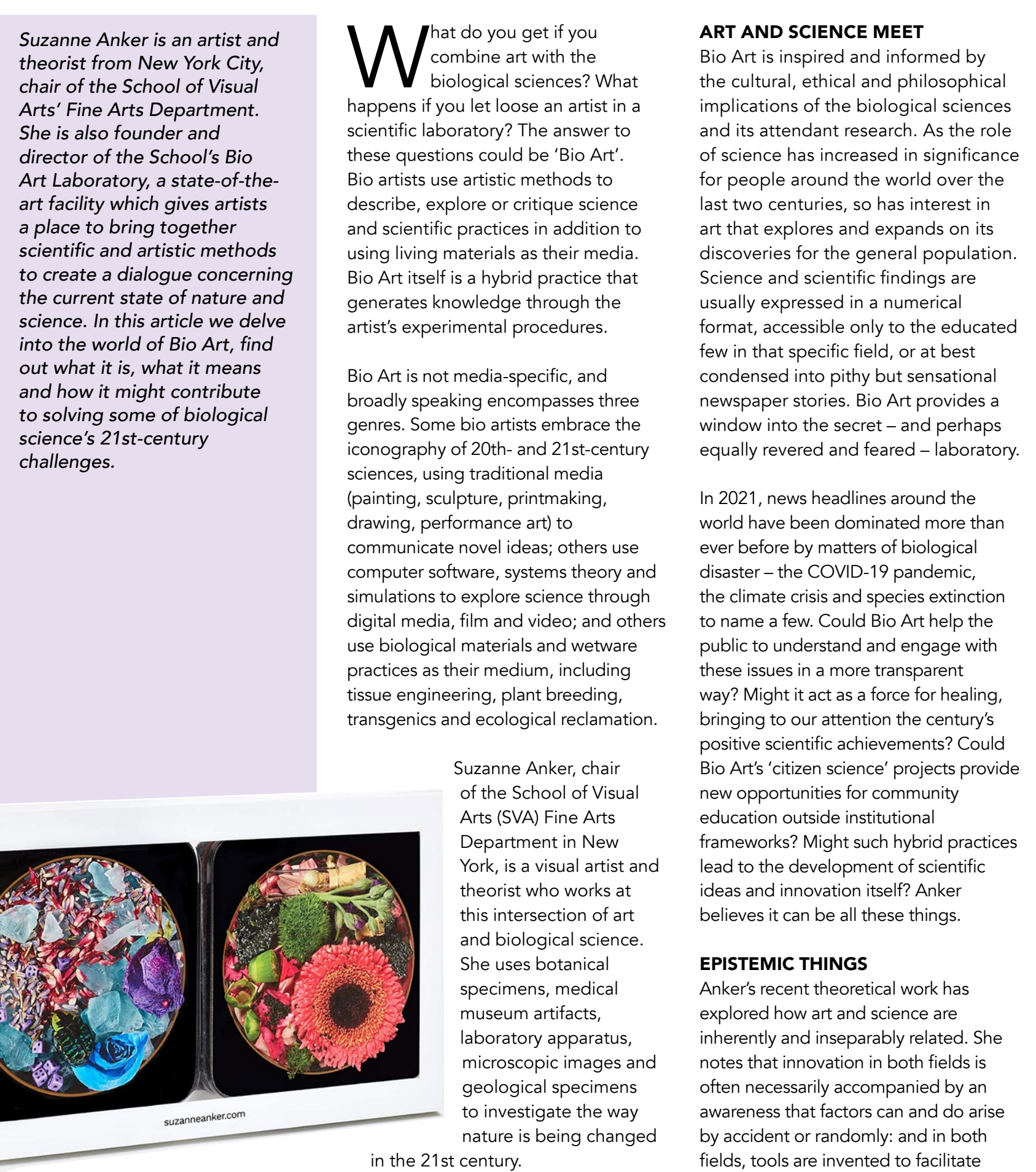

Suzanne Anker is an artist and theorist from New York City,

Art Laboratory, a state-of-the-

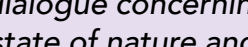

to solving some of biological

science's 21 st
challenges.

in the 21 st century.

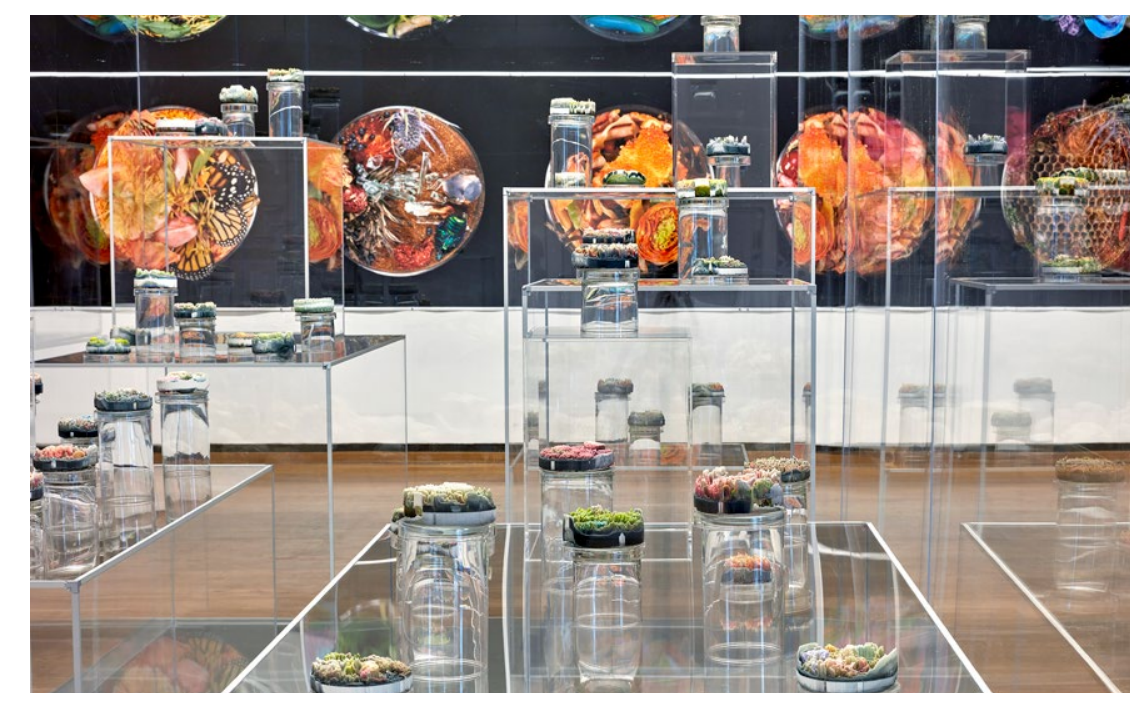

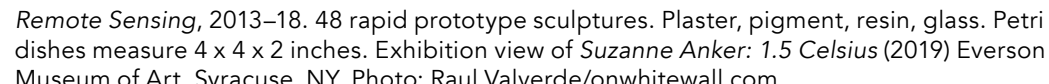

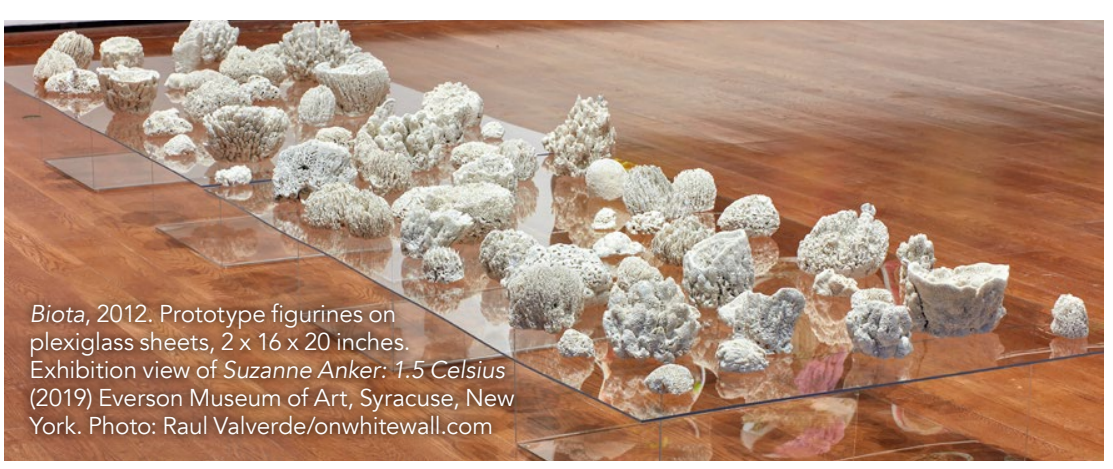

Bio Art is inspired and informed by the cultural, ethical and philosophical implications of biological science and research.

unique working methods. Anker is interested in these tools or 'epistemic generate knowledge in experimental systems. She cites as examples electrophoresis and polymerase chain reaction - techniques that have revolutionised genetic research and become the gold standard for DNA replication. Scientific discovery relies on the development of such tools that go on to generate innovation. This in turn becomes part of the Bio Art trajectory: the tools are part of the data, creating new organisms, models andifformation, and they consequently converge with $\mathrm{B}$ Art practices.

Anker also points out the significant their ability to capture the collective imagination. They are part of the epistemic process that leads to scientific discovery: a good example is Rosalind Franklin's famous 'Photo 51', the key image of DNA's double helix which led to an accurate understanding of its example of Megaw's work to acknowledge the cultural manifestation of novel scientific methodologies. Megaw was a central figure in a group made up of textile manufacturers and crystallographers who created prints revealing the properties of insulin, haemoglobin and china clay.

THE BIO ART LAB role images play in biology, as well as

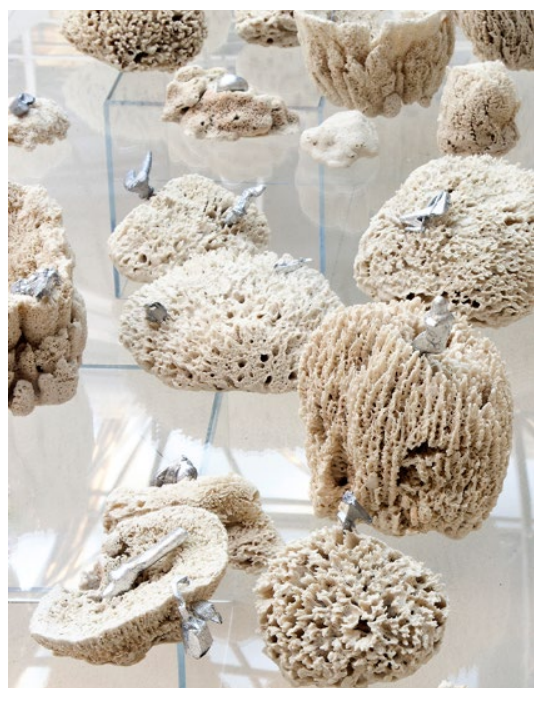

Biota, 2012. Porcelain, silver-leaf rapid prototype figurines. Exhibition view of Cereb
Spirits: Stalking the Self (2012) William Paterson University, New Je
onwhitewall.com

'scientific tools and techniques become 'sols and techniques in art practice'. The ab gives students access to equipment such as a bioprinter, microscopes, incubators, vortex mixers and more, as well as housing skeletons, a specime aquarium and library.

Some particularly culturally significan examples of Bio Art that have come out of Anker's Laboratory include Astroculture, a sculptural installation akin to an indoor garden or terrarium, in which vegetables and herbs grow metal cubes complete with indidual LED panels. Anker herself notes how environmental conditions for the plants and opens up a discussion about NASA's research into agriculture in anti-gravity conditions and the immense pressure on plant life here on Earth as a result of climate change and the death of pollinators. Could this installation contribute to the invention of the new and robust growing techniques that are now needed to ensure a stable supply of plants for both food and medicine?

Myotomato (2016) was a project consisting of wet lab practices, constructed models, video, photography and recipes, and derived tom the concept of a variety of for their large size and good taste). 


000000

The Bio Art venture engaged in a technique be used to cultivate seeds genetic engineering of the tomato's for plants that could solve malnutrition genome with myoglobin, with the in poverty-stricken or climate-chang intention of increasing the quantity was found to be present in the newly grown plants, though without further botanical expertise it was not possible dish) as household objects. Presented

to determine

Earth transform. They encourage us to take a moment to contemplate our own mortality as did the Durch still ife painters (n)

\section{ALTERING NATURE}

Our societies and culture are already

which parts of the The artwork opens up a discussion about undits as a result plant carried the

its limitations,
however, NASA's research into agriculture in anti-gravity conditions.

Myotomato clearly as coasters, these colourful and vivid expressions of life and death show flowers, insects and fruit vanishing over time - a simple warning of the delicacy
of life and the way the elements of the low-carbon, protein-rich foods could enable us to reduce the meat production hat contributes so much to many countries' carbon footprints. Could such

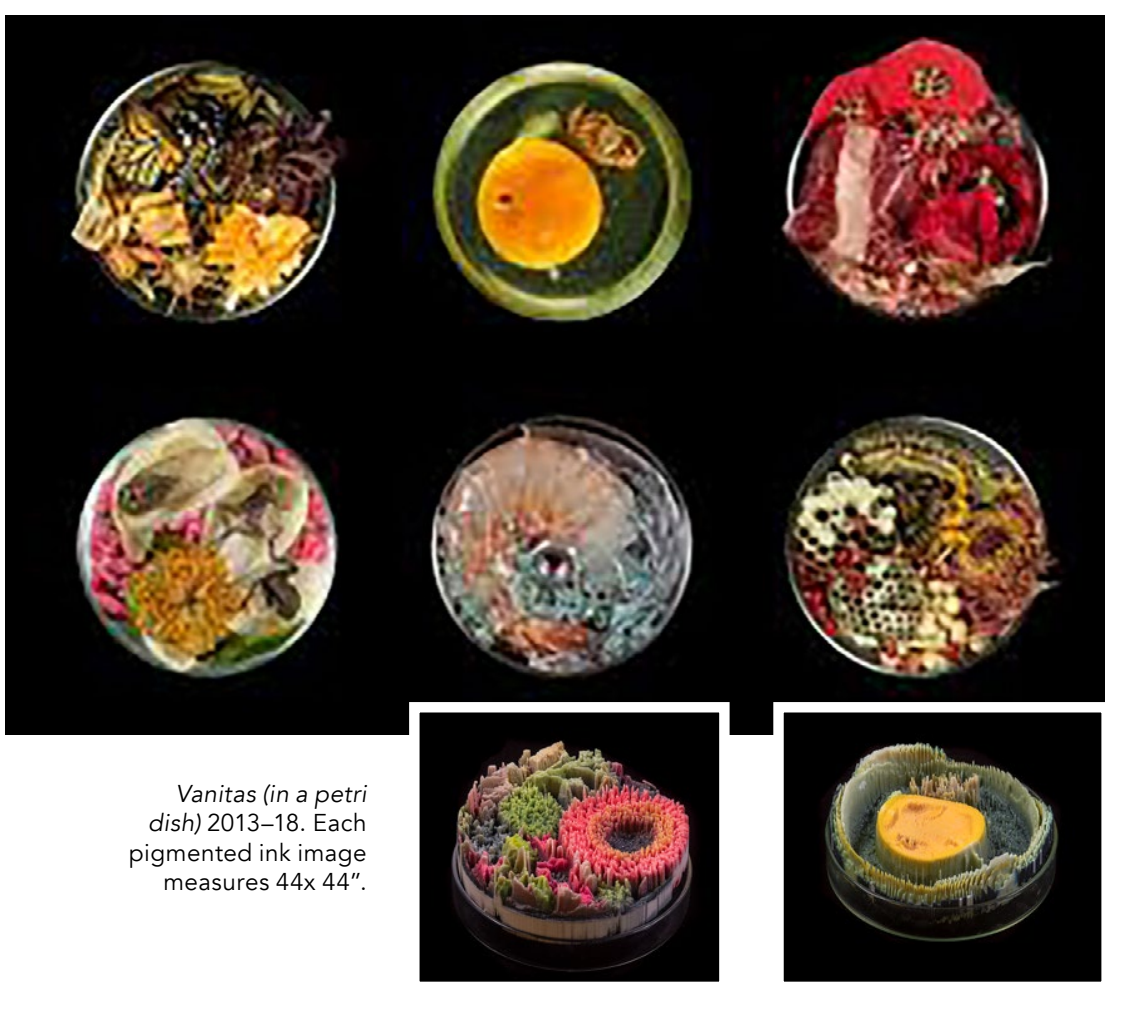
and collapsing ecosystems. Bio Art is ind and encouraging discussion of how nature is being changed in the Anthropocene an informal name given to the most have begun to make significant changes have begun to make significant changes

Prots

Projects from the Bio Art Lab, as well as Anker's own work, demonstrate that such art not only starts conversations, brings better understanding of science to the public and captures the cultural imagination, but also actively contributes to research and research and science have a special relationship their union can produce questions, (hen change the future. available on Amazon: www.amazon.com/Suzanne-AnkerVanitas-Coasters-Yellow/dp/BoovivifV7 Vanitas-Petri-Coasters/dp/B08V23BJS8/

\section{Behind the Research}

Suzanne Anker

E: suzanne.anker@gmail.com T: +1 6465492904 W: www.suzanneanker.com

Research Objectives

Suzanne uses visual art to investigate the ways in whic nature is being altered in the 21 st century.

\section{Detail}

Suzanne Anker

20 Quarty Circle, East Hampton, NY 11937

Bio

Suzanne Anker is a visual artist and theorist working at the intersection of art and the biological sciences. extinction and toxic degradation, she calls attention to the beauty of life and the 'necessity for enlightened thinking about nature's "tangled bank"

\section{SVA䌊 NYC}

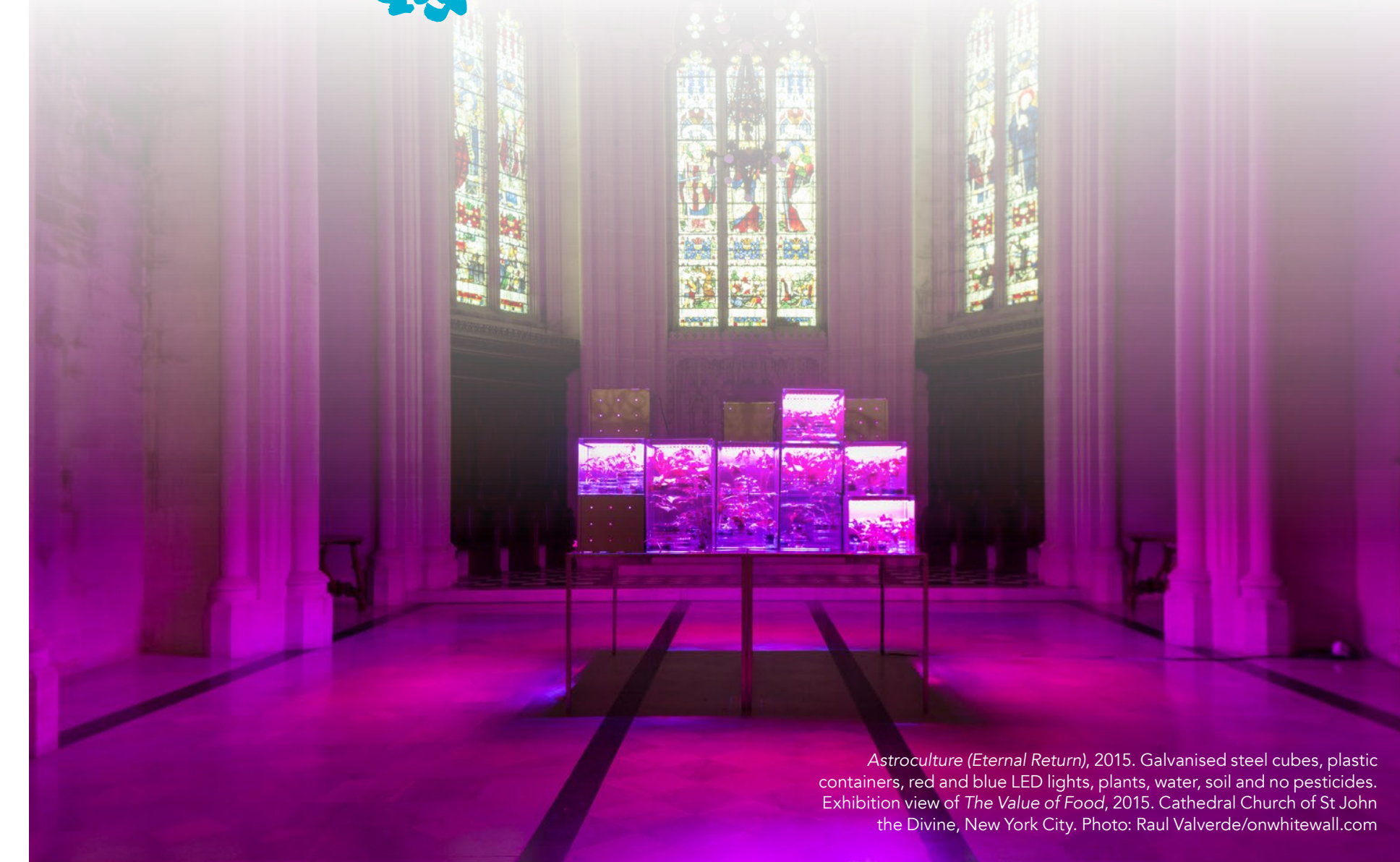

\section{References}

Yetisen, A.K., et al. (2015) Bioart. Trends in Biotechnology, [online] 33(12), 724-734. doi.org/10.1016/j. tibtech.2015.09.011

Anker, S., (2021) Epistemic practices in Bio Art. Al \& Society, doi.org/10.1016/j.tibtech.2015.09.01

\section{Personal Response}

What ideas and processes are behind Vanitas (in a

II My Vanitas (in a Petri dish) series speak to the flux of life and the regenerative powers of nature in a precarious time. In transforming my art into a wider audience, by bringing attention to the urgent
with

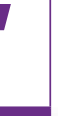

